Can. J. Math. Vol. 50 (3), 1998 pp. 487-496

\title{
ON THE LIOUVILLE PROPERTY FOR DIVERGENCE FORM OPERATORS
}

\author{
MARTIN T. BARLOW
}

\begin{abstract}
In this paper we construct a bounded strictly positive function $\sigma$ such that the Liouville property fails for the divergence form operator $L=\nabla\left(\sigma^{2} \nabla\right)$. Since in addition $\Delta \sigma / \sigma$ is bounded, this example also gives a negative answer to a problem of Berestycki, Caffarelli and Nirenberg concerning linear Schrödinger operators.
\end{abstract}

1. Introduction. In a paper on the qualitative properties of solutions of non-linear PDE of the form $\Delta u+F(u)=0$, Berestycki, Caffarelli and Nirenberg posed the following problem. (See [BCN, Theorem 1.7]).

PRoblem 1. Let $V$ be a smooth bounded function on $\mathbb{R}^{d}$, and let $K=K[V]$ be the (Schrödinger) operator

$$
K=-\Delta-V .
$$

Suppose that a bounded and sign-changing solution $u$ exists to $K u=0$. Set

$$
\lambda_{1}(K)=\inf \left\{\int_{\mathbb{R}^{d}}|\nabla \psi|^{2}-V|\psi|^{2}: \psi \in C_{0}^{\infty},\|\psi\|_{2}=1\right\} .
$$

Then is $\lambda_{1}(K)<0$ ?

[BCN, Theorem 1.7] proved that if $d=1$ or 2 then the answer to Problem 1 is "yes". In [GG] Ghoussoub and Gui proved that the answer is "no" if $d \geq 7$, and made explicit the connection (implicit in the proof of [BCN, Theorem 1.7]) between Problem 1 and the following question on the Liouville property for divergence form operators.

PROBLEM 2. Let $\sigma$ be a strictly positive $C^{2}$ function on $\mathbb{R}^{d}$, and let $L=L[\sigma]$ be the divergence form operator $L=\nabla\left(\sigma^{2} \nabla\right)$. Let $\psi$ be a solution to $L \psi=0$. If $\sigma \psi$ is bounded, then is $\psi$ constant? (If this is the case we will say that $L$ has the Liouville property).

It is well-known that if $\sigma$ is uniformly bounded away from 0 (so that $\sigma>\epsilon>0$ ) then $L[\sigma]$ has the Liouville property. The proof of [BCN, Theorem 1.7] implies that the answer to Problem 2 is "yes" if $d=1,2$, while [GG] give an example which proves that the answer to Problem 2 is "no" if $d \geq 7$. In those spaces to which the answer to Problem 1 is "yes" this result provides a powerful technique for the study of non-linear PDE-see [GG].

Received by the editors January 8, 1998; revised March 5, 1998.

Research partially supported by a NSERC (Canada) grant.

AMS subject classification: Primary: 31C05; secondary: 60H10, 35J10.

(c) Canadian Mathematical Society 1998. 
To see the connection between the two problems note first that if $\sigma>0$ is $C^{2}$ then

$$
L[\sigma] \varphi=-\sigma K\left[-\sigma^{-1} \Delta \sigma\right](\sigma \varphi) .
$$

THEOREM 1 ([GG, PRoposition 2.3, Lemma 2.1]). Let $V$ be smooth and bounded.

(a) If a bounded non-zero $C^{2}$ solution $u$ to $K[V] u=0$ exists, then $\lambda_{1}(K[V]) \leq 0$.

(b) $\lambda_{1}(K[V])<0$ if and only if $K[V] u=0$ has no positive solutions.

TheOREM 2. (See [GG, Proposition 2.8], [BCN, Theorem 1.7]).

(a) Let $V$ be bounded and smooth, and suppose a bounded sign-changing solution $u$ to $K[V] u=0$ exists. If $\lambda_{1}(K[V])=0$ then the equation $K[V] \sigma=0$ has positive solutions, and for any positive solution $\sigma$ the Liouville property fails for $L[\sigma]$.

(b) Let $\sigma>0$ be smooth, and such that $V=-\sigma^{-1} \Delta \sigma$ is bounded. Suppose there exists a sign-changing function $\varphi$ such that $\sigma \varphi$ is bounded, and $L[\sigma] \varphi=0$. Then there exists a sign-changing solution $u$ to $K[V] u=0$, but $\lambda_{1}(K[V])=0$.

Proof. (a) If $K, u, \sigma$ are as above, set $\varphi=u / \sigma$. By (1.1) $L[\sigma] \varphi=0$, while $\varphi$ is sign-changing, and therefore non-constant.

(b) Set $u=\sigma \varphi$ : by (1.1) $u$ is a bounded sign-changing solution to $K[V] u=0$. So, by Theorem 1 (a) $K[V] \leq 0$. On the other hand since $\sigma>0$ also satisfies $K[V] \sigma=0$, by Theorem 1(b) $\lambda_{1}(K[V])=0$.

REMARKS. 1. The proof above is given in [GG], but is included here for completeness.

In this paper we give an example which shows that the answer to Problems 1 and 2 is "no" for $d \geq 3$. In view of Theorem 2 we can concentrate on the Liouville property, and seek a bounded function $\sigma>0$ such that $\Delta \sigma / \sigma$ is bounded, but $L[\sigma]$ has non-trivial bounded harmonic functions. Our intuition and proofs are probabilistic. Associated with $\frac{1}{2} L[\sigma]$ is a diffusion process $\tilde{X}=\left(\tilde{X}_{t}, t \geq 0, \mathbb{P}^{x}, x \in \mathbb{R}^{d}\right)$, such that $\frac{1}{2} L[\sigma] \varphi=0$ if and only if $\varphi\left(\tilde{X}_{t}\right)$ is a $\mathbb{P}^{x}$-martingale for all $x \in \mathbb{R}^{d}$. (For accounts of the connection between elliptic operators and diffusions see for example the books [Bas], [RW]). Suppose that there exist open disjoint regions $D_{1}, D_{2}$ in $\mathbb{R}^{d}$ such that if $G_{i}=\left\{\tilde{X}_{t} \in D_{i}\right.$ for all sufficiently large $\left.t\right\}$ then

(1.2) $0<\psi_{i}(x)=\mathbb{P}^{x}\left(\tilde{X}_{t} \in D_{i}\right.$ for all sufficiently large $\left.t\right)<1, \quad i=1,2$,

for some (and so all) $x \in \mathbb{R}^{d}$. Then since $\psi_{i}$ are bounded and harmonic (with respect to $L$ ), by the martingale convergence theorem

$$
\psi_{i}\left(\tilde{X}_{t}\right) \rightarrow I_{G_{i}} \quad \text { as } t \rightarrow \infty, \mathbb{P}^{x}-\text { a.s. }
$$

Thus $\psi_{i}$ are non-constant, and it is easy to construct from them a bounded sign-changing $L$-harmonic function: $\psi=\psi_{1}-\psi_{2}$, for example.

For the regions $D_{i}$ we will take $D_{1}=\left\{x \in \mathbb{R}^{d}: x_{1}>0\right\}, D_{2}=\left\{x: x_{1}<0\right\}$. If we take $\sigma$ small in a neighbourhood of $\left\{x_{1}=0\right\}$ this creates a (partial) barrier to the process $\tilde{X}$ crossing between the regions $D_{1}$ and $D_{2}$ : note that $\tilde{X}$ satisfies the SDE

$$
d \tilde{X}_{t}=\sigma\left(\tilde{X}_{t}\right)^{2} d \tilde{B}_{t}+\sigma\left(\tilde{X}_{t}\right) \nabla \sigma\left(\tilde{X}_{t}\right) d t
$$


where $\tilde{B}$ is a $d$-dimensional Brownian motion. If $\sigma(x) \rightarrow 0$ sufficiently fast as $|x| \rightarrow \infty$ on the set $\left\{x: x_{1}=0\right\}$, then this barrier is strong enough so that $\tilde{X}$ only crosses between the regions $D_{i}$ a finite number of times, a.s. (More precisely, with probability 1 there are only finitely many $n$ such that $\tilde{X}_{t}$, crosses between the regions $D_{i}$ between times $n$ and $n+1)$. The fact that $\tilde{X}$ is transient is of course crucial here. So $\mathbb{P}\left(G_{1} \cup G_{2}\right)=1$, while $G_{1} \cap G_{2}=\emptyset$, and this, (with symmetry) proves (1.2) for $x=0$.

THEOREM 3. (a) Let $d \geq 3$. There exists a smooth strictly positive bounded function $\sigma$ on $\mathbb{R}^{d}$ such that $V=-\sigma^{-1} \Delta \sigma$ is bounded, and the equation $\nabla\left(\sigma^{2} \nabla \varphi\right)=0$ has a bounded sign-changing solution $\varphi$.

(b) If $K=-\Delta-V$, then $K u=0$ has a bounded sign changing solution $u$, and $\lambda_{1}(K)=0$.

In Section 2 we collect together some (mainly standard) properties of Bessel processes and related diffusions, and in Section 3 we give the construction of the function $\sigma$.

We use $c_{i}$ to denote fixed positive real constants, whose value only depends on the dimension $d$, and $c, c^{\prime}$ etc. to denote positive constants (depending only on $d$ ) whose value may change from line to line. We write $x \in \mathbb{R}^{d}$ as $x=\left(x_{1}, x^{(1)}\right)$, where $x^{(1)}=$ $\left(x_{2}, \ldots, x_{d}\right) \in \mathbb{R}^{d-1}$. All the functions on $\mathbb{R}^{d}$ in this paper will depend on $x$ only through $u=x_{1}, y=\left|x^{(1)}\right| . \lambda_{d}$ denotes $d$-dimensional Lebesgue measure, and $a \wedge b=\min (a, b)$.

2. Some preliminary estimates. We begin by collecting some estimates on Bessel processes and related potentials.

LEMMA 2.1. Let $d \geq 3$ and $X$ be $a \operatorname{Bes}(d)$ process. Then

$$
\mathbb{P}^{x}\left(X_{s} \leq y \text { for some } s \geq t\right) \leq t^{-1 / 2} y .
$$

PROOF. Using a comparison theorem for SDEs (see [IW, p. 353]) we can assume that $x=0$ and $d=3$. By Pitman's decomposition [P] we can write $X_{t}=2 M_{t}-B_{t}$, where $B_{t}$ is a one-dimensional Brownian motion with $B_{0}=0$, and $M_{t}=\sup _{s \leq t} B_{s}$. Then $\inf _{s \geq t} X_{s}=M_{t}$. By the reflection principle $\mathbb{P}\left(B_{t}^{+}>y\right)=2 \mathbb{P}\left(B_{t}>y\right)=\mathbb{P}\left(\left|B_{t}\right|>y\right)$, so

$$
\mathbb{P}^{x}\left(X_{s} \leq y \text { for some } s \geq t\right)=\mathbb{P}\left(\left|B_{t}\right| \leq y\right) \leq 2 y t^{-1 / 2}(2 \pi)^{-1 / 2}<t^{-1 / 2} y .
$$

LEMMA 2.2. Let $U_{t}$ be a 1-dimensional diffusion with generator $L f(u)=$ $\frac{1}{2}\left(\sigma^{2}(u) f^{\prime}(u)\right)^{\prime}$, where $\sigma(u)>\varepsilon>0$. If $0<x<y$ then

$$
\mathbb{P}^{x}(U \text { hits } 0 \text { before } y)=\frac{\Phi(x)}{\Phi(0)},
$$

where $\Phi(x)=\int_{x}^{y} \sigma^{-2}(u) d u$.

PROOF. Writing $\varphi(x)=\mathbb{P}^{x}(U$ hits 0 before $y)$, we have that $L \varphi=0$, so that $\varphi^{\prime}(x)=$ $-c \sigma^{-2}(x)$. Since $\varphi(0)=1, \varphi(y)=0,(2.2)$ follows. 
Let $G$ be the usual Green operator on $\mathbb{R}^{d}$, given by

$$
G \mu(x)=\int\left|x-x^{\prime}\right|^{2-d} \mu\left(d x^{\prime}\right),
$$

where $\mu$ is a measure on $\mathbb{R}^{d}$. Set

$$
J(a, r)=\left\{x=\left(x_{1}, x^{(1)}\right):\left|x_{1}\right| \leq a, r-a \leq\left|x^{(1)}\right| \leq r+a\right\} .
$$

LEMMA 2.3. Let $\nu$ be Lebesgue measure restricted to $J(a, r)$. Then $G \nu$ is symmetric in $x_{1}$, and $x_{1}\left(\partial G \nu / \partial x_{1}\right) \leq 0$. Also $G \nu$ depends on $x^{(1)}$ only through $y=\left|x^{(1)}\right|$. If $r \geq$ $\max \left(4 a, a^{2}\right)$ then there exist constants $c_{1}, c_{2}$ such that

$$
\begin{gathered}
c_{1} a^{2} \leq G \nu(x) \leq c_{2} a^{2} \quad \text { if }|x|<\frac{1}{2} r, \\
c_{1} a^{2} \log r \leq G \nu(x) \leq c_{2} a^{2} \log r \quad \text { if } x \in J(a, r), \\
G \nu(x) \leq c_{2} a^{2}(|x| / r)^{2-d} \quad \text { if }|x|>2 r .
\end{gathered}
$$

PROOF. The first two properties of $G \nu$ are clear from the definition and the symmetry of $J$.

We have $c a^{2} r^{d-2} \leq \nu(J(a, r)) \leq c^{\prime} a^{2} r^{d-2}$, and $\frac{3}{4} r \leq|x| \leq \frac{3}{2} r$ for $x \in J(a, r)$. So if $\left|x^{\prime}\right| \leq \frac{1}{2} r, c a^{2} \leq G \nu\left(x^{\prime}\right) \leq c^{\prime} a^{2}$, proving (2.3).

Let $x \in J(a, r)$. Then

$$
G \nu(x)=\int_{J}\left|x-x^{\prime}\right|^{2-d} d x^{\prime} \geq \int_{J \cap B(x, 2 a)^{c} \cap B(x, r)}\left|x-x^{\prime}\right|^{2-d} d x^{\prime} .
$$

If $a<s<r$ then $\lambda_{d-1}(\partial B(x, s) \cap J) \geq c a^{2} s^{d-3}$, so that

$$
G \nu(x) \geq \int_{a}^{r} c a^{2} s^{-1} d s=c a^{2} \log (r / a) .
$$

Also, if $r \geq a^{2}$ then $\log (r / a) \geq \log r^{1 / 2}=\frac{1}{2} \log r$. A similar calculation proves the other bound in (2.4).

For (2.5), since $\left|x-x^{\prime}\right| \geq \frac{1}{2}|x|$ for $x^{\prime} \in J$, and $|x|>2 r$, we have

$$
G \nu(x) \geq c a^{2} r^{d-2}\left(\frac{1}{2}|x|\right)^{2-d} \geq c^{\prime} a^{2}(|x| / r)^{2-d} .
$$

Now set $n_{k}=e^{2^{k}}, a_{k}=2^{k+1}$, and let $J_{k}=J\left(a_{k}, n_{k}\right)$ for $k \geq 0$. Set $A=\bigcup_{k=3}^{\infty} J_{k}$.

PROPOSITION 2.4. There exists $\varphi>0$ on $\mathbb{R}^{d}$ with the following properties.

(a) $\varphi$ is superharmonic, and $\Delta \varphi=0$ on $A^{c}$.

(b) $\varphi \geq 1$ on $A$.

(c) $x_{1} \partial \varphi / \partial x_{1}>0$.

(d) $\varphi$ depends on $x$ only through $u=x_{1}, y=\left|x^{(1)}\right|$. 
(e) If $\gamma(t)$ is any path in $\mathbb{R}^{d}$ such that $\lim _{\sup } \rightarrow \infty|\gamma(t)|=\infty$ then $\liminf _{t \rightarrow \infty} \varphi(\gamma(t))=0$.

PROOF. Let $\nu_{k}$ be Lebesgue measure restricted to $J_{k}$, and

$$
\varphi_{k}=c_{1}^{-1} a_{k}^{-2}\left(\log n_{k}\right)^{-1} G \nu_{k} .
$$

By Lemma 2.3 we have $\varphi_{k} \geq 1$ on $J_{k}$, and $\varphi_{k}(x) \leq c\left(\log n_{k}\right)^{-1}=c 2^{-k}$, provided $|x| \leq \frac{1}{2} n_{k}$. Set

$$
\varphi(x)=\sum_{k=3}^{\infty} \varphi_{k}(x) .
$$

Clearly $0<\varphi(x)<\infty$ for all $x$. Since each $\varphi_{k}$ is superharmonic, and harmonic on $J_{k}^{c}, \varphi$ clearly satisfies (a) and (b). (c) and (d) follow from the corresponding property for $G \nu_{k}$.

To prove (e), let $x_{k} \in \mathbb{R}^{d}$ be such that $\left|x_{k}\right|=\frac{1}{2} n_{k+1}$. Then by Lemma 2.3, if $i \leq k$,

$$
\varphi_{i}\left(x_{k}\right) \leq c\left(\log n_{i}\right)^{-1}\left(\left|x_{k}\right| / n_{i}\right)^{2-d} \leq c\left(2 n_{k} / n_{k+1}\right)=c^{\prime} e^{-2^{k}},
$$

while $\varphi_{i}\left(x_{k}\right) \leq c 2^{-k}$ if $i \geq k+1$. So,

$$
\varphi\left(x_{k}\right) \leq c k e^{-2^{k}}+c^{\prime} 2^{-k} .
$$

Since $|\gamma(t)|=\frac{1}{2} n_{k+1}$ for infinitely many $t$, it follows that

$$
\liminf _{t \rightarrow \infty} \varphi(\gamma(t)) \leq \liminf _{k \rightarrow \infty} \varphi\left(x_{k}\right)=0 .
$$

Let $X_{t}, t \geq 0$ be a process in $\mathbb{R}^{d}$. We define the event

$$
\{X \text { ultimately avoids } A\}=\bigcup_{n=0}^{\infty}\left\{X_{t} \notin A \text { for all } t \geq n\right\} .
$$

COROLlaRY 2.5. Let B be a Brownian motion in $\mathbb{R}^{d}$. Then $\mathbb{P}^{x}(B$ ultimately avoids A) $=1$.

PROOF. $\varphi\left(B_{t}\right)$ is a positive supermartingale, and so converges a.s. Using Proposition 2.4(e) we see that $\lim _{t \rightarrow \infty} \varphi\left(B_{t}\right)=0$ a.s. Since $\varphi(x) \geq 1$ on $A$, it follows that $B$ ultimately avoids $A$, a.s.

3. The counterexample. Let $\sigma>0, f$ be functions on $\mathbb{R}^{d}$ which depend on $x$ only through $u$ and $y$. Then if $L[\sigma]=\nabla\left(\sigma^{2} \nabla\right)$,

$$
\frac{1}{2} L[\sigma] f=\frac{1}{2} \sigma^{2}\left(f_{u u}+f_{y y}\right)+\sigma \sigma_{u} f_{u}+\left(\sigma \sigma_{y}+\sigma^{2} \frac{d-2}{2 y}\right) f_{y} .
$$

We will restrict our attention to operators on $\mathbb{R}^{d}$ of this form. Recall the definitions of $n_{k}$, $J_{k}, A$ from Section 2. For $k \geq 1$ let

$$
\bar{\sigma}_{k}(u)=1 \wedge n_{k}^{-1} e^{|u|} .
$$


Let $\bar{\sigma}(u, y)$ be given by

$$
\begin{gathered}
\bar{\sigma}(u, y)=\bar{\sigma}_{k}(u), n_{k-1}+2^{k-1} \leq y \leq n_{k}, \quad k \geq 4 \\
\bar{\sigma}(u, y)=1 \wedge \exp \left(-2^{k-1}+|u|-\left(y-n_{k-1}\right)\right), n_{k-1} \leq y \leq n_{k-1}+2^{k-1}, \quad k \geq 4 \\
\bar{\sigma}(u, y)=\bar{\sigma}_{3}(u), \quad 0 \leq y \leq n_{3}
\end{gathered}
$$

Let $\psi$ be a symmetric $C^{\infty}$ function supported on $\left(-\frac{1}{2}, \frac{1}{2}\right)$, and set

$$
\sigma_{k}(u)=\int \psi\left(u-u^{\prime}\right) \bar{\sigma}\left(u^{\prime}\right) d u^{\prime}, \quad \sigma(u, y)=\iint \psi\left(u-u^{\prime}\right) \psi\left(y-y^{\prime}\right) \sigma\left(u^{\prime}, y^{\prime}\right) d u^{\prime} d y^{\prime} .
$$

It is straightforward to verify

LEMMA 3.1. $\quad \sigma_{k}$ and $\sigma$ are bounded smooth strictly positive functions on $\mathbb{R}$ and $\mathbb{R} \times \mathbb{R}_{+}$ which satisfy:

$$
\begin{gathered}
\bar{\sigma}_{k}(u)=\bar{\sigma}(-u), \quad \sigma(u, y)=\sigma(-u, y), \\
|\Delta \sigma| \leq c_{3} \sigma, \\
u \sigma_{u} \geq 0, \quad \sigma_{y}=0 \text { on } A^{c}, \\
\sigma(u, y)=\bar{\sigma}_{k}(u) \quad \text { if } n_{k-1}+2^{k} \leq y \leq n_{k}-2^{k+1} \\
\int_{2^{k}-1}^{2^{k}} \sigma_{k}^{-2}(u) d u \leq c_{4}, \quad \int_{0}^{1} \sigma_{k}^{-2}(u) d u \geq c_{5} n_{k}^{2} .
\end{gathered}
$$

Now let $L_{1}$ be the operator given by

$$
L_{1} f=\frac{1}{2} \sigma^{2}\left(f_{u u}+f_{y y}\right)+\sigma \sigma_{u} f_{u}+\left(\sigma \sigma_{y}+\sigma^{2} \frac{d-2}{2 y}\right) f_{y},
$$

and set $L_{2}=\sigma^{-2} L_{1}$. Let $Z_{t}=\left(\left(U_{t}, Y_{t}\right), t \geq 0, \mathbb{P}^{z}, z \in \mathbb{R} \times \mathbb{R}_{+}\right)$be the diffusion associated with $L_{2}$. Then $Z$ is (the unique) solution to the $\mathrm{SDE}$

$$
\begin{gathered}
d U_{t}=d B_{t}+\left(\frac{\sigma_{u}\left(Z_{t}\right)}{\sigma\left(Z_{t}\right)}\right) d t, \\
d Y_{t}=d B_{t}^{\prime}+\left(\frac{\sigma_{y}\left(Z_{t}\right)}{\sigma\left(Z_{t}\right)}+\frac{d-2}{2 Y_{t}}\right) d t,
\end{gathered}
$$

where $B, B^{\prime}$ are independent one-dimensional Brownian motions. Write $g(u, y)=$ $\sigma_{u}(u, y) / \sigma(u, y)$ : by (3.7) $g \geq 0$. Set $V_{t}=U_{t}^{2}$ : then by Itô's formula

$$
\begin{aligned}
d V_{t} & =2 V_{t}^{1 / 2} \operatorname{sgn}\left(U_{t}\right) d B_{t}+\left(1+2 V_{t}^{1 / 2} g\left(V_{t}^{1 / 2}\right)\right) d t \\
& =2 V_{t}^{1 / 2} d \bar{B}_{t}+\left(1+2 V_{t}^{1 / 2} g\left(V_{t}^{1 / 2}\right)\right) d t .
\end{aligned}
$$

Here

$$
\operatorname{sgn}(x)= \begin{cases}0 & \text { if } x \leq 0 \\ 1 & \text { if } x>0\end{cases}
$$


and $\bar{B}_{t}$, given by

$$
\bar{B}_{t}=\int_{0}^{t} \operatorname{sgn}\left(U_{t}\right) d B_{t},
$$

is another one-dimensional Brownian motion—see [RW, p. 63]. Let $\bar{V}$ be the solution to

$$
d \bar{V}_{t}=2 \bar{V}_{t}^{1 / 2} d \bar{B}_{t}+d t, \quad \bar{V}_{0}=V_{0} .
$$

By a comparison theorem for SDEs (see [IW, p. 353]) it follows that $\bar{V}_{t} \leq V_{t}=U_{t}^{2}$ for all $t \geq 0$. However, (3.13) implies that $\bar{V}^{1 / 2}$ is a Bes(1) process, and so equal in law to the absolute value of a Brownian motion. (See [RW, p. 69]).

Set

$$
\begin{gathered}
T_{A}=\inf \left\{t \geq 0:\left(U_{t}, Y_{t}\right) \in A\right\}, \\
\bar{T}_{A}=\inf \left\{t \geq 0:\left(\bar{V}_{t}^{1 / 2}, Y_{t}\right) \in A\right\} .
\end{gathered}
$$

We have $\bar{T}_{A} \leq T_{A}$. From (3.7) and (3.11) we deduce that if $\bar{Y}$ is the solution to

$$
d \bar{Y}_{t}=d B_{t}^{\prime}+\frac{d-2}{2 \bar{Y}_{t}} d t, \quad \bar{Y}_{0}=Y_{0},
$$

then $\bar{Y}$ is a $\operatorname{Bes}(d-1)$ process, and $\bar{Y}_{t}=Y_{t}$ for $0 \leq t \leq T_{A}$. Let also $\bar{Z}_{t}=\left(U_{t}, \bar{Y}_{t}\right)$, and $\bar{R}_{t}=\left(\bar{V}_{t}+\bar{Y}_{t}^{2}\right)^{1 / 2}$ : then $\bar{R}$ is a $\operatorname{Bes}(d)$ process, and $\left|\bar{Z}_{t}\right| \geq \bar{R}_{t}$.

Now set

$$
\begin{gathered}
H_{k}(t)=\left\{(u, y): n_{k-1}+2^{k} \leq y \leq n_{k}-2^{k+1},|u|=t\right\}, \quad k \geq 4, \\
I_{k}=\left[-2^{k}, 2^{k}\right] \times\left[n_{k-1}+2^{k}, n_{k}-2^{k+1}\right], \quad k \geq 4, \\
H_{3}(t)=\left\{(u, y): 0 \leq y \leq n_{3},|u|=t\right\} .
\end{gathered}
$$

Fix $k \geq 4$ and define stopping times $S_{i}, T_{i}$ by

$$
\begin{gathered}
T_{0}=0, \\
S_{n}=\inf \left\{t \geq T_{n-1}: Z_{t} \in H_{k}\left(2^{k}-1\right)\right\}, \\
T_{n}=\inf \left\{t \geq S_{n}: Z_{t} \in H_{k}(0) \cup H_{k}\left(2^{k}\right) \cup A\right\} .
\end{gathered}
$$

Note that $Z_{t} \in I_{k}$ for $S_{n} \leq t \leq T_{n}$, and that if $Z$ hits $H_{k}(0)$ and $T_{A}=\infty$ then $Z_{T_{n}} \in H_{k}(0)$ for some $n$.

LEMMA 3.2. On $\left\{S_{n}<\infty\right\}$,

$$
\mathbb{P}^{z}\left(Z_{T_{n}} \in H_{k}(0), T_{n}<T_{A} \mid \mathcal{F}_{S_{n}}\right) \leq c n_{k}^{-2} .
$$

PROOF. Using the Markov property of $Z$, we can assume $n=1$ and $S_{1}=0, Z_{0}=$ $\left(u_{0}, y_{0}\right) \in H_{k}\left(2^{k}-1\right)$. On $0 \leq t \leq T_{1}$ we therefore have that $U$ satisfies the SDE

$$
U_{t}=u_{0}+B_{t}+\int_{0}^{t} g_{k}\left(U_{s}\right) d s,
$$


where $g_{k}=\sigma_{k}^{-1} \partial \sigma_{k} / \partial u$. If $U^{\prime}$ is the solution to (3.16) for $0 \leq t<\infty$, then $U=U^{\prime}$ on $\left[0, T_{1}\right]$. Set $T^{\prime}=\inf \left\{t: U_{t}^{\prime} \in\left\{0,2^{k}\right\}\right\}$. So

$$
\begin{aligned}
\mathbb{P}\left(U_{T_{1}}=0, T_{1}<T_{A}\right) & =\mathbb{P}\left(U_{T_{1}}^{\prime}=0, T_{1}<T_{A}\right) \\
& \leq \mathbb{P}\left(U_{T^{\prime}}=0\right) \\
& \leq \int_{2^{k}-1}^{2^{k}} \sigma_{k}^{-2}(u) d u / \int_{0}^{2^{k}} \sigma_{k}^{-2}(u) d u \leq c_{6} n_{k}^{-2} .
\end{aligned}
$$

Here we used Lemma 2.2 and the estimate (3.9) in the last line.

Now set

$$
t_{k}=4^{k} n_{k}^{2}, \quad m_{k}=k t_{k}^{1 / 2}=k 2^{k} n_{k} .
$$

LEMMA 3.3. On $\left\{T_{n-1}<\infty\right\} \cap\left\{T_{n-1}<T_{A}\right\} \cap\left\{\left|U_{T_{n-1}}\right| \geq 2^{k}\right\}$

$$
\mathbb{P}^{z}\left(S_{n}-T_{n-1}>t_{k} \mid \mathcal{F}_{T_{n-1}}\right) \geq c_{7} t_{k}^{-1 / 2}
$$

PROOF. As in the previous proof, it is enough to obtain the estimate for $S_{1}-T_{0}$ in the case when $Z_{0}=\left(u_{0}, y_{0}\right) \in H_{k}\left(2^{k}\right)$. Using the comparison between $U_{t}$ and $\bar{V}_{t}^{1 / 2}$ we have

$$
\mathbb{P}\left(S_{1}-T_{0}>t_{k}\right) \geq \mathbb{P}\left(T_{-1}(\beta)>t_{k}\right),
$$

where $\beta$ is a one-dimensional Brownian motion started at 0 , and $T_{-1}(\beta)=\inf \left\{s: \beta_{s}=\right.$ -1 . However using the reflection principle as in Lemma 2.1,

$$
\mathbb{P}\left(T_{-1}(\beta)>t\right)=\mathbb{P}\left(\left|B_{t}\right|<1\right) \sim c t^{-1 / 2}, \quad \text { as } t \rightarrow \infty .
$$

Set

$$
\begin{gathered}
N_{k}=\max \left\{n: S_{n}<\infty\right\}, \\
G=\left\{U_{T_{n}}=0 \text { for some } n \leq m_{k} \wedge N_{k}\right\}, \\
\eta=\max _{1 \leq n \leq N_{k} \wedge m_{k}}\left(S_{n}-T_{n-1}\right),
\end{gathered}
$$

Then if $z \notin I_{k}$ and $k \geq 4$,

$$
\begin{aligned}
\mathbb{P}^{z}(Z \text { hits } & \left.H_{k}(0), T_{A}=\infty\right) \\
= & \mathbb{P}^{z}\left(Z \text { hits } H_{k}(0), G, T_{A}=\infty\right)+\mathbb{P}^{z}\left(Z \text { hits } H_{k}(0), G^{c}, T_{A}=\infty\right) . \\
& \leq \mathbb{P}^{z}\left(G, T_{A}=\infty\right)+\mathbb{P}^{z}\left(N_{k}>m_{k}, G^{c}, T_{A}=\infty\right)
\end{aligned}
$$

By Lemma 3.2 the first term in (3.17) is bounded by $c_{2} m_{k} n_{k}^{-2}$. If $T_{A}=\infty$, then $Z=\bar{Z}$, and so $\left|Z_{t}\right| \geq \bar{R}_{t}$ for all $t$. We have

$$
\mathbb{P}\left(N_{k}>m_{k}, G^{c}, T_{A}=\infty\right)=\mathbb{P}\left(N_{k}>m_{k},\left|U_{T_{n}}\right|=2^{k} \text { for } 1 \leq n \leq m_{k}, \eta<t_{k}, T_{A}=\infty\right)
$$

$$
+\mathbb{P}\left(N_{k}>m_{k}, G^{c}, \eta \geq t_{k}, T_{A}=\infty\right) \text {. }
$$


The first term in (3.18) is bounded by

(3.19) $\mathbb{P}\left(N_{k}>m_{k}, S_{n}-T_{n-1}<t_{k}\right.$ for $\left.1 \leq n \leq m_{k}, G^{c}, T_{A}=\infty\right) \leq\left(1-c_{7} t_{k}^{-1 / 2}\right)^{m_{k}}$,

by Lemma 3.3. If $N_{k}>m_{k}$ and $\eta \geq t_{k}$ then $Z_{t_{0}} \in H_{k}\left(2^{k}-1\right)$ for some $t_{0}>t_{k}$. Since $\left|Z_{t_{0}}\right|^{2} \leq\left(2^{k}-1\right)^{2}+n_{k}^{2} \leq 4 n_{k}^{2}$, we deduce from (2.1) that

$\mathbb{P}^{z}\left(N_{k}>m_{k}, G^{c}, \eta \geq t_{k}, T_{A}=\infty\right) \leq \mathbb{P}^{z}\left(\overline{R_{t}}<2 n_{k}\right.$ for some $\left.t \geq t_{k}\right) \leq 2 t_{k}^{-1 / 2} n_{k}$.

Collecting these estimates together, we have

$$
\begin{aligned}
\mathbb{P}^{z}\left(Z \text { hits } H_{k}(0), T_{A}=\infty\right) & \leq c m_{k} n_{k}^{-2}+\left(1-c_{7} t_{k}^{-1 / 2}\right)^{m_{k}}+2 t_{k}^{-1 / 2} n_{k} \\
& \leq c k 2^{k} n_{k}^{-1}+e^{-c_{7} k}+2^{1-k}=\varepsilon_{k},
\end{aligned}
$$

where $\sum_{k=2}^{\infty} \varepsilon_{k}<\infty$.

LEMMA 3.4. (a) Z ultimately avoids A, a.s.

(b) $Z$ is transient.

(c) For any $z \in \mathbb{R} \times \mathbb{R}_{+}$,

$\mathbb{P}^{z}\left(Z\right.$ hits $H_{k}(0)$ for infinitely many $\left.k, T_{A}=\infty\right)=0$.

PROOF. (a) From the properties of the function $\varphi$ in Proposition 2.4, we see that if $\bar{\varphi}(u, y)$ is the function such that $\varphi(x)=\bar{\varphi}(u(x), y(x))$, then $u \bar{\varphi}_{u} \geq 0$. Since on $A^{c} \bar{\varphi}$ satisfies

$$
\frac{1}{2}\left(\bar{\varphi}_{u u}+\bar{\varphi}_{y y}\right)+\frac{d-2}{2 y} \bar{\varphi}_{y}=0,
$$

we have on $A^{c}$

$$
L_{2} \bar{\varphi}=\sigma^{-1} \sigma_{u} \varphi_{u} \leq 0 .
$$

So $1 \wedge \bar{\varphi}\left(Z_{t}\right)$ is a supermartingale, and so converges a.s. to some limit. But since $\left|Z_{t}\right| \geq$ $\left|U_{t}\right| \geq \bar{V}_{t}^{1 / 2}$, and lim $\sup _{t \rightarrow \infty} \bar{V}_{t}^{1 / 2}=\infty$, by Proposition 2.4(e) we have that the limit must be 0 . Thus, as in Corollary 2.5, $Z$ ultimately avoids $A$.

(b) This is immediate from (a).

(c) Since $z$ is in at most one of the sets $I_{k}$, this is immediate from the estimate (3.20) and the Borel-Cantelli lemma.

THEOREM 3.5. Z Z ultimately avoids $\{u=0\}, \mathbb{P}^{z}$-a.s.

ProOF. Since $\mathbb{P}^{z}(Z$ ultimately avoids $A)=1$, we have

$$
0=\lim _{n \rightarrow \infty} \mathbb{P}^{z}\left(Z_{t} \in A \text {, for some } t \geq n\right)=\lim _{n \rightarrow \infty} \mathbb{E}^{z}\left(\mathbb{P}^{Z_{n}}\left(T_{A}<\infty\right)\right) .
$$

Note that $\{u=0\} \subseteq \Gamma=A \cup \bigcup_{k=3}^{\infty} H_{k}(0)$. Set $F_{n}=\left\{Z_{t} \in \Gamma\right.$ for some $\left.t \geq n\right\}$, $F=\bigcap_{n=0}^{\infty} F_{n}$. Then

$$
\mathbb{P}^{z}(F)=\mathbb{P}^{z}\left(F \cap\left\{T_{A}<\infty\right\}\right)+\mathbb{P}^{z}\left(F \cap\left\{T_{A}=\infty\right\}\right) .
$$


If $F$ occurs then either $Z$ hits infinitely many of the $H_{k}(0)$, or $Z$ hits one of the components of $\Gamma$ after time $n$ for infinitely many $n$. But as $Z$ is transient the second possibility has probability 0 . So

$$
\mathbb{P}^{z}\left(F \cap\left\{T_{A}=\infty\right\}\right)=\mathbb{P}^{z}\left(Z \text { hits } H_{k}(0) \text { for infinitely many } k, T_{A}=\infty\right)=0
$$

by Lemma 3.4(c).

So,

$$
\mathbb{P}^{z}(F)=\mathbb{P}^{z}\left(F \cap\left\{T_{A}<\infty\right\}\right) \quad \text { for } z \in \mathbb{R} \times \mathbb{R}_{+}
$$

But

$$
\mathbb{P}^{z}(F)=\mathbb{E}^{z}\left(\mathbb{P}^{Z_{n}}(F)\right)=\mathbb{E}^{z}\left(\mathbb{P}^{Z_{n}}\left(F \cap\left\{T_{A}<\infty\right\}\right)\right) \leq \mathbb{E}^{z} \mathbb{P}^{Z_{n}}\left(T_{A}<\infty\right),
$$

which converges to 0 as $n \rightarrow \infty$ by (3.21). So $\mathbb{P}^{z}(F)=0$.

By Theorem 3.5 we see that if $D_{1}=\{u>0\}, D_{2}=\{u<0\}$ and $G_{i}=\left\{Z_{t} \in D_{i}\right.$ for all sufficiently large $t\}$, then $G_{1} \cap G_{2}=\emptyset$, while $\mathbb{P}^{z}\left(G_{1} \cup G_{2}\right)=1$. By symmetry $\mathbb{P}^{0}\left(G_{i}\right)=\frac{1}{2}$. Set $\psi_{i}(z)=\mathbb{P}^{z}\left(G_{i}\right)$. We have $\psi_{1}+\psi_{2}=1,0<\psi_{i}<1$ and since $\psi_{i}\left(Z_{t}\right)$ is a martingale, by the martingale convergence theorem $\psi_{i}\left(Z_{t}\right) \rightarrow I_{G_{i}}$ a.s., which shows that $\psi_{i}$ are non-constant. So $\psi=\psi_{1}-\psi_{2}$ is a sign-changing function which is harmonic with respect to the operator $L_{2}$. Hence $L_{1} \psi=\sigma^{2} L_{2} \psi=0$. We have proved:

COROLLARY 3.5. The equation $L_{1} \psi=0$ has a bounded sign-changing solution.

PROOF OF THEOREM 3. Recall the notation $x=\left(x_{1}, x^{(1)}\right), u=x_{1}, y=\left|x^{(1)}\right|$. Let $\sigma$, $\psi$ be as above, and define $\tilde{\sigma}(x)=\sigma(u(x), y(x)), \tilde{\psi}(x)=\psi(u(x), y(x))$. Then $\tilde{\sigma}$ and $\tilde{\sigma}^{-1} \Delta \tilde{\sigma}$ are bounded, and

$$
L[\tilde{\sigma}] \tilde{\psi}=2 L_{1} \psi=0,
$$

so that $\tilde{\psi}$ is a bounded sign-changing solution of $\nabla\left(\tilde{\sigma}^{2} \nabla \tilde{\psi}\right)=0$. The final assertion in Theorem 3 is now immediate from Theorem 2.

\section{REFERENCES}

[Bas] R. F. Bass, Diffusions and Elliptic Operators. Springer, New York, 1997.

[BCN] H. Berestycki, L. Cafarelli and L. Nirenberg, Further qualitative properties for elliptic equations in unbounded domains. preprint.

[GG] N. Ghoussoub and C. Gui, On a conjecture of De Giorgi and some related problems. Math. Ann., to appear.

[IW] N. Ikeda and S. Watanabe, Stochastic Differential Equations and Diffusion processes. North-Holland, Kodansha, 1981.

[P] J. Pitman, One dimensional Brownian motion and the three-dimensional Bessel process. J. Appl. Probab. 7(1975), 511-526.

[RW] L. C. G. Rogers and D. Williams, Diffusions, Markov Processes, and Martingales, Volume I. (2nd edition), Wiley, 1994.

Department of Mathematics University of British Columbia Vancouver, British Columbia V6T $1 Z 2$ 